\title{
Fractional flow reserve-guided coronary artery bypass grafting: Can intraoperative physiologic imaging guide decision making?
}

\author{
T. Bruce Ferguson, Jr, MD, Cheng Chen, PhD, Joseph D. Babb, MD, Jimmy T. Efird, PhD, \\ Ramesh Daggubati, MD, and John M. Cahill, MD
}

\begin{abstract}
Objectives: Fractional flow reserve-guided coronary artery bypass grafting is emerging in cardiac surgery, in which the nature (anatomic and functional characteristics) of the target vessel epicardial coronary artery stenosis is important in graft site selection. The nature of the stenosis might determine a different physiologic response to bypass grafting. We report our recent experience using near infrared fluorescence complex angiography and perfusion analysis to identify the nature of stenoses in the target vessel by imaging the physiologic response to grafting.
\end{abstract}

\begin{abstract}
Methods: In 167 patients who underwent consecutive multivessel coronary artery bypass grafting cases (63\% off-pump coronary artery bypass grafting) with traditional anatomy-based revascularization, we imaged and analyzed 359 grafts (53\% arterial). This platform provides angiographic data of both the target vessel epicardial coronary artery and graft simultaneously (to assess the imaged competitive flow); and because a change in fluorescence intensity is proportional to the change in blood flow and perfusion, the quantified change (if any) in regional myocardial perfusion surrounding the grafted target vessel epicardial coronary artery.
\end{abstract}

Results: The patient outcomes in our series were excellent. All 359 grafts were widely patent by angiography, and $24 \%$ of the arterial and $22 \%$ of the saphenous vein grafts showed no regional myocardial perfusion change in response to bypass grafting. In 165 in situ internal mammary artery grafts to the left anterior descending artery ( $>70 \%$ stenosis), 40 had no change in regional myocardial perfusion, and 32 of the 40 had competitive flow imaged.

Conclusions: An important number of angiographically patent bypass grafts demonstrated no change in regional myocardial perfusion, suggesting anatomic, but nonfunctional, stenoses in those target vessel epicardial coronary arteries. In in situ arterial grafts, imaged competitive flow is associated with nonfunctional stenoses in the target vessel epicardial coronary artery. Imaging these physiologic responses to target vessel revascularization might be useful in the emerging fractional flow reserve-guided era. (J Thorac Cardiovasc Surg 2013;146:824-35)

Video clip is available online.

Supplemental material is available online.

\footnotetext{
From the Department of Cardiovascular Sciences, East Carolina Heart Institute, Brody School of Medicine at East Carolina University, Greenville, NC.

Disclosures: Drs Ferguson and Chen have been consultants to Novadaq Technologies, Inc, which manufactures the SPY Near-Infrared Angiography system for cardiac surgery use. Drs Ferguson and Chen invented, copyrighted, and patented the CAPA Platform with Novadaq Technologies, Inc. The other authors have nothing to disclose with regard to commercial support.

Read at the 93rd Annual Meeting of The American Association for Thoracic Surgery, Minneapolis, Minnesota, May 4-8, 2013.

Received for publication May 7, 2013; revisions received June 1, 2013; accepted for publication June 18, 2013; available ahead of print Aug 5, 2013.

Address for reprints: T. Bruce Ferguson, Jr, MD, Department of Cardiovascular Sciences, East Carolina Heart Institute, Brody School of Medicine at East Carolina University, 115 Heart Dr, Greenville, NC 27834 (E-mail: fergusont@ecu.edu). $0022-5223 / \$ 36.00$

Copyright $\odot 2013$ by The American Association for Thoracic Surgery http://dx.doi.org/10.1016/j.jtcvs.2013.06.026
}

For 50 years, the conceptual and practical basis of surgical revascularization with coronary artery bypass grafting $(\mathrm{CABG})$ has been the underlying coronary anatomy ${ }^{1}$ and the accompanying stenotic ${ }^{2}$ atherosclerotic plaque and/or thrombotic occlusion in the target vessel epicardial coronary artery (TVECA). Early on, incomplete revascularization within this anatomy-based construct was associated with a $15 \%$ reduction in 5 -year survival, ${ }^{3}$ and the principle of complete anatomic revascularization became linked to these anatomic stenotic triggers.

Using this approach, the outcomes from isolated CABG have improved dramatically, despite the significant increase in preoperative risk of patients undergoing CABG. ${ }^{4,5}$ The 5 -year results from the Synergy between Percutaneous Coronary Intervention with Taxus and Cardiac Surgery ${ }^{6}$ (SYNTAX) and Future Revascularization Evaluation in Patients with Diabetes Mellitus: Optimal Management of Multivessel Disease ${ }^{7}$ (FREEDOM) trials, along with observational database analyses, have documented that in certain patient populations, CABG is a preferable treatment alternative to multivessel percutaneous coronary intervention (PCI), because a long-term mortality benefit is conveyed with $\mathrm{CABG}^{8}$ 


\begin{tabular}{|c|c|}
\hline \multicolumn{2}{|c|}{ Abbreviations and Acronyms } \\
\hline CABG & $=$ coronary artery bypass grafting \\
\hline CAPA & $\begin{aligned}= & \text { complex angiography and } \\
& \text { perfusion analysis }\end{aligned}$ \\
\hline COURAGE & $\begin{aligned}= & \text { Clinical Outcomes Utilizing } \\
& \text { Revascularization and Aggressive } \\
& \text { Drug Evaluation }\end{aligned}$ \\
\hline $\mathrm{CPB}$ & $=$ cardiopulmonary bypass \\
\hline FAME & $\begin{aligned}= & \text { Fractional Flow Reserve Versus } \\
& \text { Angiography for Multivessel } \\
& \text { Disease }\end{aligned}$ \\
\hline FFR & $=$ fractional flow reserve \\
\hline FREEDOM & $\begin{aligned} &= \text { Future Revascularization } \\
& \text { Evaluation in Patients with } \\
& \text { Diabetes Mellitus: Otpimal } \\
& \text { Management of Multivessel } \\
& \text { Disease }\end{aligned}$ \\
\hline ICF & $=$ image-described competitive flow \\
\hline ICG & $=$ indocyanine green \\
\hline IDAP & $=$ image data acquisition protocol \\
\hline IDS & $=$ image data sequence ( 34 seconds) \\
\hline IMA & $=$ internal mammary artery \\
\hline NIRF & $=$ near-infrared fluorescence \\
\hline OPCAB & $=$ off-pump CABG \\
\hline PCI & $\begin{aligned} &= \text { percutaneous coronary } \\
& \text { intervention }\end{aligned}$ \\
\hline PREVENT-I & $\begin{aligned} I= & \text { Project of Ex-vivo Vein Graft } \\
& \text { Engineering via Transfection IV }\end{aligned}$ \\
\hline RA & $=$ radial artery \\
\hline RMP & $=$ regional myocardial perfusion \\
\hline SVG & $=$ saphenous vein graft \\
\hline SYNTAX & $\begin{array}{l}=\text { Synergy between Percutaneous } \\
\text { Coronary Intervention with Taxus } \\
\text { and Cardiac Surgery }\end{array}$ \\
\hline TVECA & $\begin{array}{l}=\text { target vessel epicardial coronary } \\
\text { artery }\end{array}$ \\
\hline
\end{tabular}

In parallel, relentless advancement in our understanding of patients with chronic stable angina in need of revascularization continues. The Clinical Outcomes Utilizing Revascularization and Aggressive Drug Evaluation 9 (COURAGE) trial established the importance of optimal medical therapy for these patients, and a substudy identified the requirement of a significant $(>10 \%)$ degree of myocardial ischemia for revascularization strategies to have clinical benefit. ${ }^{10}$ In a paradigm shift, fractional flow reserve (FFR) analysis of anatomic lesions has transformed an anatomic stenosis to a "functional stenosis" classification. ${ }^{11}$ In such cases, the anatomic lesion is presumptively linked to regional ischemia and/or a perfusion deficit in the surrounding myocardium supplied by the TVECA. In the Fractional Flow Reserve Versus Angiography for Multivessel Disease (FAME) trial, ${ }^{12}$
$20 \%$ of the anatomic lesions with $71 \%$ to $90 \%$ stenosis and $60 \%$ of lesions with $51 \%$ to $70 \%$ had no measured functionality. In the FAME 2 trial, ${ }^{13}$ many patients with more severe 3 -vessel anatomic disease were reclassified as having 2- or 1 -vessel functional disease.

Thus, surgical revascularization is at the point at which anatomy as the sole criterion for the revascularization strategy needs to be reconsidered. We report a unique, real-time intraoperative imaging technology to identify differences in the physiologic (angiographic and functional) response to revascularization, on a per graft basis. These physiologic findings could be critically important in a FFR-guided revascularization strategy adapted for CABG.

\section{METHODS}

\section{Imaging Technology Developments}

Near-infrared fluorescence (NIRF) angiography in CABG has been previously described, with mixed results. ${ }^{14-18}$ The fidelity of NIRF versus conventional angiography was inferior, but the technique was better at identifying potential anastomotic technical issues than transittime flowmetry. ${ }^{19}$

NIRF uses the nontoxic fluorophore indocyanine green (ICG) dye, administered as a bolus injection into the blood stream. ${ }^{20,21}$ The pharmacokinetics of its binding to endothelial cells and circulating proteins, its metabolism by the liver, and excretion by the kidneys is well-understood, with a half-time of approximately 90 seconds. The fluorophore is excited by a low-energy NIR laser, and the fluorescence image data are collected as a 34-second image data sequence (IDS) of 1020 images at a camera speed of 30 frames/s. Because no radiation is involved, this full 34-second image data set can be safely captured with each ICG injection. ICG fluorescence behavior in the heart has been studied in nearly 1000 patients, and we have confirmed clinically the experimental data validating that the ICG behavior is consistent on the first pass through the coronary arteries; the fluorescence intensity is proportional to the concentration of dye and dose administered and to the circulating blood volume; and under certain conditions, the regional fluorescence intensity is directly proportional to the myocardial blood flow and myocardial perfusion. ${ }^{22}$ Therefore, a change in fluorescence intensity will be a direct indicator of a corresponding change in myocardial perfusion.

Early on, we recognized that the 34-second image data sequence contained considerably more information about the myocardial blood flow and perfusion than did angiography alone. We developed, tested, and implemented a complex angiography and perfusion analysis (CAPA) platform into the NIRF system for real-time intraoperative analysis.

\section{Clinical Experience With NIRF-CAPA}

From May 2009 (when the image data acquisition protocol [IDAP] for the post- versus pregrafting comparison with the CAPA platform was validated) through March 2013 (to allow for full 30-day follow-up), we performed 167 consecutive isolated $\mathrm{CABG}$ procedures with $\geq 2$ grafts placed. All patients underwent CABG by 1 of us (T.B.F., Jr), using predetermined revascularization strategy-based anatomy, with a revascularization trigger of $\geq 70 \%$ for TVECAs and $>50 \%$ for left main disease.

We attempted to image all the grafts in all patients using this standardized IDAP and platform. Technical reasons were the cause for nonimaged patients and/or grafts; this diminished with accrued experience. All patient clinical characteristics were captured in a combined American College of Cardiology/Society of Thoracic Surgeons clinical database for analysis.

The raw IDS and the NIRF-CAPA analysis data were stored in a unique data construct that directly combined the imaging data with the clinical data; thus, access to the raw imaging data files was always available for 
subsequent review and analysis and for batch analysis processing of the image analysis algorithms over time during their development.

\section{Statistical Analysis}

The institutional review board at East Carolina University approved the present longitudinal study.

The clinical data are expressed as continuous or discrete variables as mean values and percentages. The Society of Thoracic Surgeons benchmarks from the latest available analysis (quarter 3, 2012) were used for comparison of the clinical demographics and outcomes. Histogram plots were assessed by comparing the slope of the log exponential decay curves. $P<.05$ was considered significant.

\section{RESULTS \\ Clinical Data Set}

The clinical population demographics, procedural characteristics, and outcomes of the patients undergoing revascularization (Table 1) were consistent with those of contemporary surgical revascularization populations reported recently. 5,23

The results for these patients were, in general, better than the national benchmarks. Also, the consistent use of the NIRF-CAPA platform did not create any unforeseen complications or adverse effects in our population.

\section{NIRF Imaging and CAPA Platform}

Figure 1, $A$, is a single frame from the IDS, highlighting the angiographic characteristics of the TVECA and graft. Video 1 illustrates the entire 34-second IDS of this in situ left internal mammary artery (IMA) to left anterior descending coronary artery graft. The NIR spectrum is beyond the visible wavelengths; thus, the standard image presentation is a 1 to 255 gray scale image.

We adapted and developed several color presentation schemes to illustrate intuitively the image data presentation. Application of these schemes did not alter the underlying image metadata or require image processing. Figure $1, B$, shows the same graft at peak fluorescence, highlighting the perfusion to the surrounding myocardium. This is presented dynamically from this perfusion perspective in Video 2.

Figure 2 illustrates the CAPA platform analysis presentation construct and representative NIRF-CAPA analyses. Both the pre- and postgrafting image sequences, acquired some 30 seconds apart, were incorporated into the CAPA platform presentation. The IDAP is designed such that, if properly executed, the hemodynamic, coronary autoregulation, pharmacologic, and image data acquisition influences on the IDS metadata will be controlled and therefore will not influence the relative comparison analysis.

Figure 2 presents the analysis from 2 different patients undergoing in situ IMA grafting to the left anterior descending coronary artery. Both TVECAs had 75\% proximal stenoses, as assessed preoperatively. Using NIRF angiography, both grafts were widely patent, with no technical issues identified. However, a significant increase occurred in regional myocardial perfusion (RMP) as a result of bypass grafting in the patient whose data is shown in Figure 2, A, but no increase was seen in RMP in the patient with data shown in Figure 2, $B$. Using this NIRF-CAPA platform on a per graft basis, we examined the relative occurrence of RMP changes and the possible causes of these differences.

\section{Clinical and Imaging Data Set}

A total of 167 patients underwent isolated CABG (67\% urgent), with $\geq 2$ grafts (Figure 3 ). Of these patients, 7 were eliminated because technical reasons obviated a quantitative comparison. Of the 160 patients, 105 underwent standard off-pump CABG (OPCAB), and 55 underwent conventional on-pump CABG with cardioplegic arrest $(14 \%)$, or, more recently, beating heart CABG with cardiopulmonary bypass $(\mathrm{CPB})$ support at near normothermia. In the CPB patients, imaging was performed for each graft during $\mathrm{CPB}$, with stable hemodynamics and some ejection to effect normal pulsatile coronary perfusion, either after each graft or before weaning from CPB. During OPCAB, imaging was performed immediately after graft construction per the IDAP.

A total of 386 grafts were imaged in these 160 patients. The total number of grafts constructed in this group was 434 (2.7 grafts/patient); the difference was that grafts to the back of the heart in cardioplegic arrest cases were not imaging early in our experience. From these 386 imaged grafts, 359 could be analyzed using the CAPA platform. The technical problem with the 27 nonanalyzed grafts was usually related to ICG injection in the patients on $\mathrm{CPB}$; since changing this component of the IDAP, this problem has been eliminated.

Of the 359 analyzed grafts, $192(53.4 \%)$ were arterial, and $167(47 \%)$ were saphenous vein grafts (SVGs) (Figure 3). Of the arterial grafts, 165 were in situ IMA grafts and 27 were radial artery (RA) grafts.

All 359 grafts were documented in the NIRF angiographic evaluation on a per-graft basis to be widely patent; however, only 275 of the grafts $(77 \%)$ had at least a minimum $5 \%$ to $10 \%$ increase in RMP documented (Figure 3, A). The remaining 84 grafts $(23 \%)$ had no quantified increase in RMP.

In the subset of patent arterial grafts without an RMP increase (Figure 3, B), we identified specific angiographic characteristics that were then correlated with the "imaged competitive flow (ICF)." Video 3 illustrates the characteristic findings of ICF in an in situ arterial graft in which no RMP increase occurred.

Figure E1 shows a histogram plot of the relative frequencies of RMP increase, by graft category, for all 359 grafts in the 160 patients. These histogram frequency plots were curve fitted with exponential decay curves for comparison between in situ IMA and aortocoronary (SVG/RA) grafts. Comparing the log exponential decay curves, the curve for the IMA group had a longer and 
TABLE 1. Patient characteristics $(n=167)$

\begin{tabular}{|c|c|c|}
\hline Variable & $\begin{array}{l}\text { Patients } \\
\text { (n) }\end{array}$ & $\begin{array}{c}\text { STS Q3 } 2012 \\
\text { Benchmark }(\%)\end{array}$ \\
\hline \multicolumn{3}{|l|}{ Demographics } \\
\hline Age $\geq 65 \mathrm{yrs}$ & $106(63.5)$ & 53.3 \\
\hline \multicolumn{3}{|l|}{ Gender } \\
\hline Male & $122(73.1)$ & \\
\hline Female & $45(27.0)$ & 25.9 \\
\hline $\mathrm{BMI} \geq 30 \mathrm{~kg} / \mathrm{m}^{2}$ & $71(42.5)$ & \\
\hline Active smoker & $56(33.5)$ & 33.4 \\
\hline Hypertension & $150(89.8)$ & 87.3 \\
\hline Dyslipidemia & $152(91.0)$ & 86.4 \\
\hline Diabetes mellitus & $78(46.5)$ & 44.6 \\
\hline Cerebrovascular disease & $27(16.2)$ & 14.3 \\
\hline Previous CVA & $16(9.6)$ & 7.2 \\
\hline $\mathrm{RF}$, preoperative dialysis & $10(6.0)$ & 2.7 \\
\hline \multicolumn{3}{|l|}{ Coronary anatomy } \\
\hline 1-Vessel CAD & $5(3.0)$ & 4.4 \\
\hline 2-Vessel CAD & $40(24.0)$ & 19.9 \\
\hline 3-Vessel CAD & $122(73.1)$ & 75.3 \\
\hline $\mathrm{LM} \geq 50 \%$ & $44(26.4)$ & 33.0 \\
\hline \multicolumn{3}{|l|}{ Myocardial function } \\
\hline $\mathrm{EF}<40 \%$ & $30(18.0)$ & 15.5 \\
\hline NYHA class $\geq$ III & $26(15.6)$ & \\
\hline NYHA class III & $13(7.8)$ & 6.0 \\
\hline NYHA class IV & $13(7.8)$ & 3.9 \\
\hline Previous PCI & $39(23.4)$ & 28.6 \\
\hline History of MI & $112(67.1)$ & 50.5 \\
\hline \multicolumn{3}{|l|}{ Operative data } \\
\hline IMA used & $159(95.2)$ & 95.0 \\
\hline$>1$ distal arterial graft & $57(34.1)$ & \\
\hline Perioperative MI & $1(0.6)$ & \\
\hline On-pump & $62(37.1)$ & \\
\hline Beating heart & $38(22.8)$ & \\
\hline Cardioplegic arrest & $24(14.3)$ & \\
\hline OPCAB & $105(62.8)$ & 18.0 \\
\hline \multicolumn{3}{|l|}{ Status } \\
\hline Elective & $52(31.1)$ & 39.0 \\
\hline Urgent & $112(67.1)$ & 56.1 \\
\hline Emergent or salvage & $3(1.8)$ & 4.6 \\
\hline \multicolumn{3}{|l|}{ Outcomes } \\
\hline PROM & $1.9 \%$ & \\
\hline \multicolumn{3}{|l|}{$\mathrm{O} / \mathrm{E}$} \\
\hline Mortality or morbidity & {$[0.72]$} & 1.0 \\
\hline Mortality & {$[0.64]$} & 1.0 \\
\hline Reoperation & {$[0.58]$} & 1.0 \\
\hline Permanent stroke & {$[0.47]$} & 1.0 \\
\hline $\mathrm{RF}$ & {$[0.00]$} & 1.0 \\
\hline Prolonged ventilation & {$[0.76]$} & 1.0 \\
\hline Short length of stay & [1.16] & 1.0 \\
\hline Long length of stay & {$[0.76]$} & 1.0 \\
\hline $\begin{array}{l}\text { Data in parentheses are percentas } \\
\text { Thoracic Surgeons; } B M I \text {, body } \\
\text { failure; } C A D \text {, coronary artery dis } \\
N Y H A \text {, New York Heart Assoc } \\
M I \text {, myocardial infarction; IM } \\
\text { coronary artery bypass grafting; } \\
\text { expected ratio. }\end{array}$ & $\begin{array}{l}\text { in brackets ar } \\
\text {; } C V A \text {, cerebr } \\
A, \text { left main ( } \\
{ }^{\circ} C I \text {, percutan } \\
\text { al mammary } \\
\text { predicted risk }\end{array}$ & $\begin{array}{l}\text { tios. } S T S \text {, Society of } \\
\text { ar accident; } R F \text {, renal } \\
E, \text { ejection fraction; } \\
\text { ronary intervention; } \\
O P C A B \text {, off-pump } \\
\text { ality; } O / E \text {, observed/ }\end{array}$ \\
\hline
\end{tabular}

broader decay than the SVG/RA grafts, reflecting a physiologic difference between the graft conduits and the TVECA.

Figure 4 shows a 2-dimensional representation of the physiology of multivessel revascularization from 2 different patients undergoing OPCAB. In both cases, an anatomic strategy for revascularization was used, all 3 bypassed TVECAs had a minimum of $\geq 70 \%$ proximal stenoses, and all grafts were widely patent using NIRF. The RMP change was compared with the baseline pregraft status (Figure 4, white). Patient 1 had a minimal increase in the anterior and lateral perfusion areas, with a substantial twofold increase in the inferior right coronary artery distribution (Figure 4, yellow). In sharp contrast, patient 2 had substantial increases in RMP in all 3 (1.45-fold anterior, 1.6-fold inferior, and 2.8-fold lateral) regions of the heart as a result of $\mathrm{CABG}$.

\section{DISCUSSION}

Influences beyond the coronary anatomy alone can affect the physiologic consequences of $\mathrm{CABG}$, as we have demonstrated in our study for the first time.

These NIRF-CAPA platform data have demonstrated (1) consistent findings in both in situ arterial and aortocoronary arterial and vein grafts, from a representative patient experience; (2) analysis on a per graft basis; (3) how the use of standardized intraoperative imaging technology can open a critical new window into the real-time physiologic assessment of surgical revascularization; and (4) how this use can contribute to a more complete understanding of the factors that constitute reasonable incomplete revascularization $^{24}$ at $\mathrm{CABG}$.

By collating both the angiographic and the functional consequences of revascularization, unique clinical physiologic data are now available that challenge important CABG assumptions, including the anatomic construct for the CABG intervention strategy, competitive flow, and graft patency.

By illustrating in a new and fundamental way the physiology of revascularization, this NIRF-CAPA platform creates the opportunity for $\mathrm{CABG}$ to move in parallel with the recent physiology-based developments in FFRdirected PCI. Whether FFR-guided $\mathrm{CABG}^{25-27}$ will realize the same improved outcomes is a critical question for surgeons, especially because of the now well-documented long-term mortality benefit of $\mathrm{CABG}$ for patients with severe multivessel coronary artery disease.

\section{NIRF-CAPA Platform}

The NIRF-CAPA platform uniquely generates, from a single video sequence, both angiographic and functional evaluation of grafts to TVECAs. Because CABG is intended to improve myocardial perfusion, feedback on 


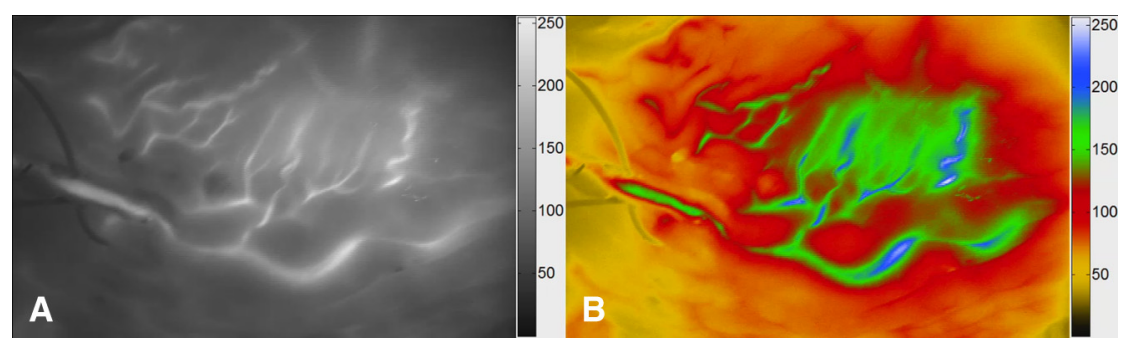

FIGURE 1. Physiologic imaging of angiography and perfusion with near-infrared fluorescence complex angiography and perfusion analysis. A, A single frame at near-peak fluorescence from the 1020 frames captured in each 34-second video sequence with near-infrared fluorescence angiography of an in situ internal mammary artery to the left anterior coronary artery graft. The raw data are presented as a 1 to 255 grey scale because the near-infrared spectrum is outside the visible spectrum. This grey scale best illustrates the angiographic component of the dynamic image data. B, The same single frame at near-peak fluorescence. These data are presented to highlight perfusion rather than angiography. This color scheme presents alternative warm and cold colors to facilitate the visual differentiation between levels of fluorescence, which represent differences in levels of perfusion. Note that no manipulation of the raw image metadata is required to generate these displays.

both the angiographic findings and the physiology of perfusion is important to assess the full benefit of the surgical revascularization.

NIRF technology was approved by the Food and Drug Administration for use in cardiac surgery in 2005, with CAPA platform development occurring during the previous 6 years. This has included the careful evaluation of a number of intraoperative, hemodynamic, physiologic, pharmacologic, and metabolic factors that could influence the imaging analysis results.

The critical importance of the IDAP was validated by beta testing of the CAPA software at centers worldwide. Because the measurement of perfusion is dependent on the conditions at measurement, the NIRF-CAPA IDAP (90 to $100 \mathrm{~s} / \mathrm{graft}$ ) generates a relative post- to pregraft comparison in which the differences in conditions that could affect perfusion and the measured change are minimal. This approach was readily adaptable to both on-pump and off-pump per graft analyses. Because bypass grafting augments blood flow to the TVECA myocardium if the myocardium can physiologically respond to the additional blood flow, when a change in perfusion is identified, it will reflect resolution from a deficiency status. Our study results have indicated that using the anatomic criteria, $75 \%$ to $80 \%$ of grafts will provide this resolution, which might be the intraoperative documentation of the relief of the preoperative ischemia or perfusion deficit seen with FFR. The per graft analysis metadata can be aggregated to illustrate the global physiologic effect of surgical revascularization compared with the preoperative, TVECA perfusion baseline (Figure 4).

\section{Per Graft Imaging and Analysis}

The present study's findings address, with this real-time physiologic data and analysis, 3 assumptions about bypass grafts to TVECAs that have developed over the years.

The nature of stenoses in TVECAs. The first assumption is that the anatomic stenosis is the only preoperative consideration important in surgical revascularization decision-making.
However, a failure to increase RMP in angiographically patent grafts ( $24 \%$ arterial, $22 \% \mathrm{SVGs}$ ) suggests nonfunctionality of the TVECA stenosis compared with the a priori expectation that all grafts will improve blood flow and perfusion. This incidence was similar to the $20 \%$ occurrence of nonfunctionality seen in lesions with angiographic anatomic stenoses between $71 \%$ and $90 \%$ in FAME $1 .{ }^{12}$

In an early prospective study applying FFR to pre-CABG patients, Botman et $\mathrm{al}^{28}$ correlated the 1-year graft patency to the corresponding stenosis functionality. Revascularization was determined using the traditional anatomic strategy, and the surgeon was unaware of the preoperative FFR results. At 1 year, $8.9 \%$ of the bypass grafts to functional TVECAs were occluded, and $21.4 \%$ of the grafts to nonfunctional stenoses were occluded. On retrospective comparison, 168 of the 525 grafts ( $32 \%$ ) were to TVECAs with nonfunctional stenoses by FFR. This percentage was slightly greater than the incidence of negative RMP change in the present study $(22 \%$ to $24 \%)$. However, in the study by Botman et al, ${ }^{28}$ the cutoff of significance for FFR was $\leq 0.75$, and intermediate anatomic lesions (50\% to $70 \%$ stenosis) were grafted.

Finally, the similarity of the RMP percentage of change in both in situ IMA and aortocoronary grafts in Figure 3 suggests a common underlying influence in both graft categories, namely the nature of the TVECA stenosis. These data, in aggregate, support the consideration that the nature of the TVECA stenosis in CABG is important. Imaged competitive flow. A second assumption relates to the competitive flow between the TVECA and the graft conduit. Anatomic proximal lesion stenosis has been the surrogate for competitive flow in most studies, ${ }^{29,30}$ but the findings from the present study suggest other influences are important.

One important angiographic advantage of this platform compared with all other evaluation technologies is that both the native TVECA and the bypass conduit can be imaged at the same time, under physiologic conditions. 
This allows for the visual identification of the dynamic interaction between the 2 conduits in real time.

The subset of in situ IMA grafts without an increase in RMP helped determine the importance of the ICF in such circumstances. In in situ IMA grafts, the association between no change in RMP and ICF was high $(80 \%)$. Of the 165 IMA grafts, 42 had evidence of ICF on the NIRF-CAPA angiographic evaluation, and $94 \%$ of these ICF grafts had no change in RMP.

This opportunity to characterize ICF both angiographically and functionally (Video 3) will substantially facilitate better understanding of the effects of ICF on the flow dynamics and graft patency. These in situ arterial graft findings are additional evidence that the functionality of the proximal stenosis at $\mathrm{CABG}$ might be as important as suggested by the FAME studies. Clearly, the etiology and significance of "competitive flow" is more complex than the percentage of anatomic stenosis alone and must also account for the functionality of the stenosis.

In contrast, as shown in Figure 3, ICF has not been identified in SVGs and in only 1 RA graft, thus far. Additional investigation is ongoing, and the difference might be related to the conduit size and the hemodynamics of aortocoronary blood flow versus native epicardial coronary flow versus in situ IMA flow.

This constellation of angiographic patency, no ICF, and no increase in RMP for "unknown reasons" occurred more frequently in the aortocoronary conduits than in the in situ IMA conduits. The data shown in Figure E1 suggest a subtle, but significant, difference between these 2 conduit types, in which an increase in RMP occurs. These data highlight that an assumption that bypass grafting beyond "critical stenoses" provides a physiologic improvement to the patient's heart, as measured at surgery, is not always correct.

Imaging, functionality, and graft failure in CABG. The present study has also provided new information about possible causes of intermediate graft failure. Bypass grafts beyond anatomic stenoses should not affect ischemia or perfusion deficits; grafts beyond functional stenoses should provide physiologic relief of both these circumstances. In the study by Botman et $\mathrm{al},{ }^{28}$ the patency rates were significantly greater in the functional intermediate (anatomic stenoses $50 \%$ to $70 \%$ ) lesions bypassed; however, no difference was found in the angina class between patients with occluded versus patent grafts. This absence of clinical consequences from graft occlusion is similar to the findings from the Project of Ex-vivo Vein Graft Engineering via Transfection IV (PREVENT IV) study, ${ }^{31}$ in which, overall, $26 \%$ of the SVGs were occluded at protocol-specified angiography. However, the 1-year angiographic occlusion did not predict an increase in mortality or death compared with patients without graft occlusion at 5 years. ${ }^{32}$ In brief, in both studies, the grafts might have been occluded simply because no TVECA regional physiologic drive was present to keep the graft open. This would be the case if the occluded grafts were placed to TVECAs with anatomic, but not functional, stenoses.

In chronic stable ischemia, the relief of a significant $(>10 \%)$ amount of ischemia is the functional component of reasonable incomplete revascularization, as described by Dauerman ${ }^{24}$ and others. ${ }^{33,34}$ Additional studies will determine whether the NIRF-CAPA finding of increased RMP is the intraoperative correlate of the relief of significant ischemia or the relief of (and thereby evidence of) a regional myocardial perfusion deficit by FFR. Our data thus far have seemed to suggest that this might be the case, based on the distribution and nature of the bypassed stenoses in the present study. Our data have further suggested that the physiologic status of the myocardium might play a significantly important role in graft attrition.

\section{Imaging and FFR-Guided CABG}

In an FFR-guided CABG construct, TVECAs with documented functional stenoses would be bypassed. In addition, most believe that surgeons would need to bypass nonfunctional, but anatomic, stenoses (ie, $>70 \%$ proximal left anterior descending coronary artery stenoses) deemed important based on historical information or for acute surgical indications. If the consequence of this FFRguided strategy is to decrease the number of grafts performed, it would challenge the principle of complete anatomic revascularization but perhaps adds to a better understanding of reasonable incomplete revascularization.

However, at this point, the effect of these nonfunctional stenoses in PCI revascularization is much better understood than in CABG. Although we can hypothesize what might be the fate of grafts to anatomic but nonfunctional stenoses, we do not know for sure whether the consequence will be adverse (failure leading to myocardial infarction and poorer long-term survival) or beneficial ("protective" effect as long as the graft is open, contributing to the long-term mortality benefit versus PCI in the SYNTAX and FREEDOM trials). The use of intraoperative imaging to physiologically document the nature of the TVECA stenoses would help connect the preoperative data with short- and long-term outcome data, on a per graft basis.

\section{Study Limitations}

The present study had several important limitations. First, we have reported a single-center experience with the NIRF-CAPA imaging technology from the center that developed the CAPA platform. These findings must be replicated for additional validation of the analysis results. However, the consistency and scope of the present study suggest that these imaging results accurately reflect the underlying physiology of surgical revascularization. 


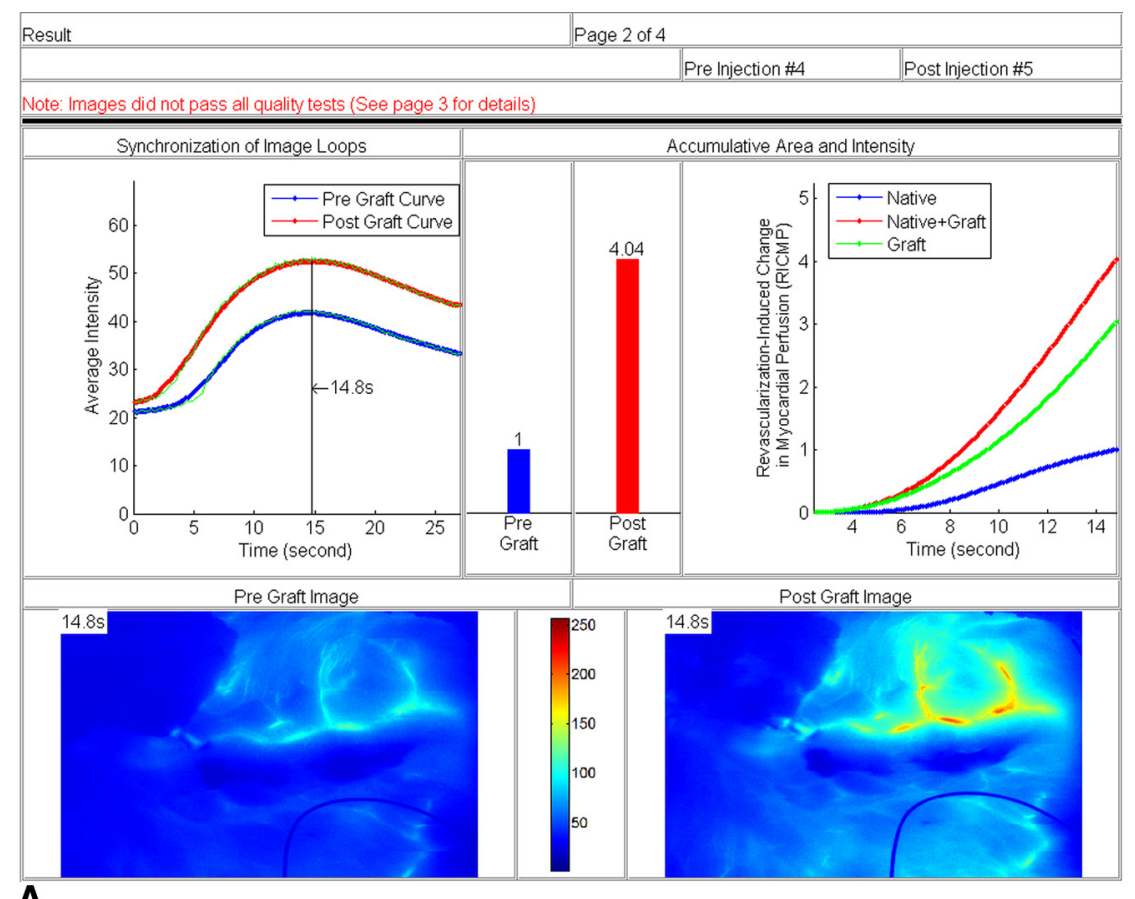

$\mathbf{A}$

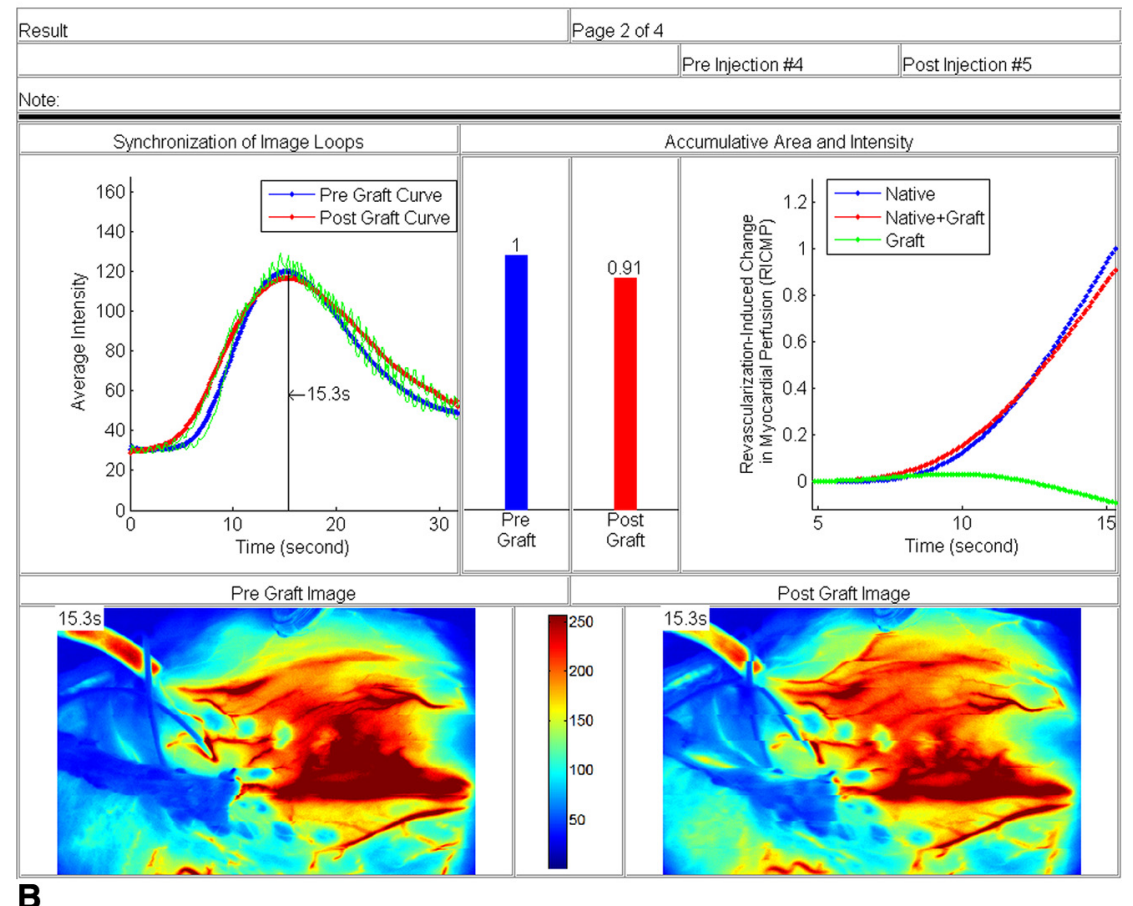

FIGURE 2. The complex angiography and perfusion analysis platform presentation construct, with representative patient analyses. Dynamic video and relative perfusion comparisons are displayed. Unique synchronization of the postgrafting (lower right) versus pregrafting (lower left) image sequences allowed for nonquantitative, but visual, comparison of these data. The complex angiography and perfusion analysis platform analyzes the underlying video metadata for the quantitative comparison of the postgrafting versus pregrafting regional myocardial perfusion in the regional territory supplied (at least in part) by the grafted target vessel epicardial coronary artery. This relative comparison is presented using 2 methods in this scheme. Upper right, The blue line represents perfusion before grafting, with only the native target vessel epicardial coronary artery supplying the myocardial region; the red line represents perfusion after grafting, with both the target vessel epicardial coronary artery and graft supplying the myocardial region; and the green line is the calculated difference in myocardial perfusion, if any, as a result of bypass grafting. Upper middle, The bar graph normalizes the pregraft perfusion to 1 and illustrates this relative comparison as a ratio against this normalized value. A and B, Analysis from 2 different patients undergoing in situ internal mammary artery grafting to the left anterior descending coronary artery. A, A significant increase occurred in regional myocardial perfusion in the anterior wall. 


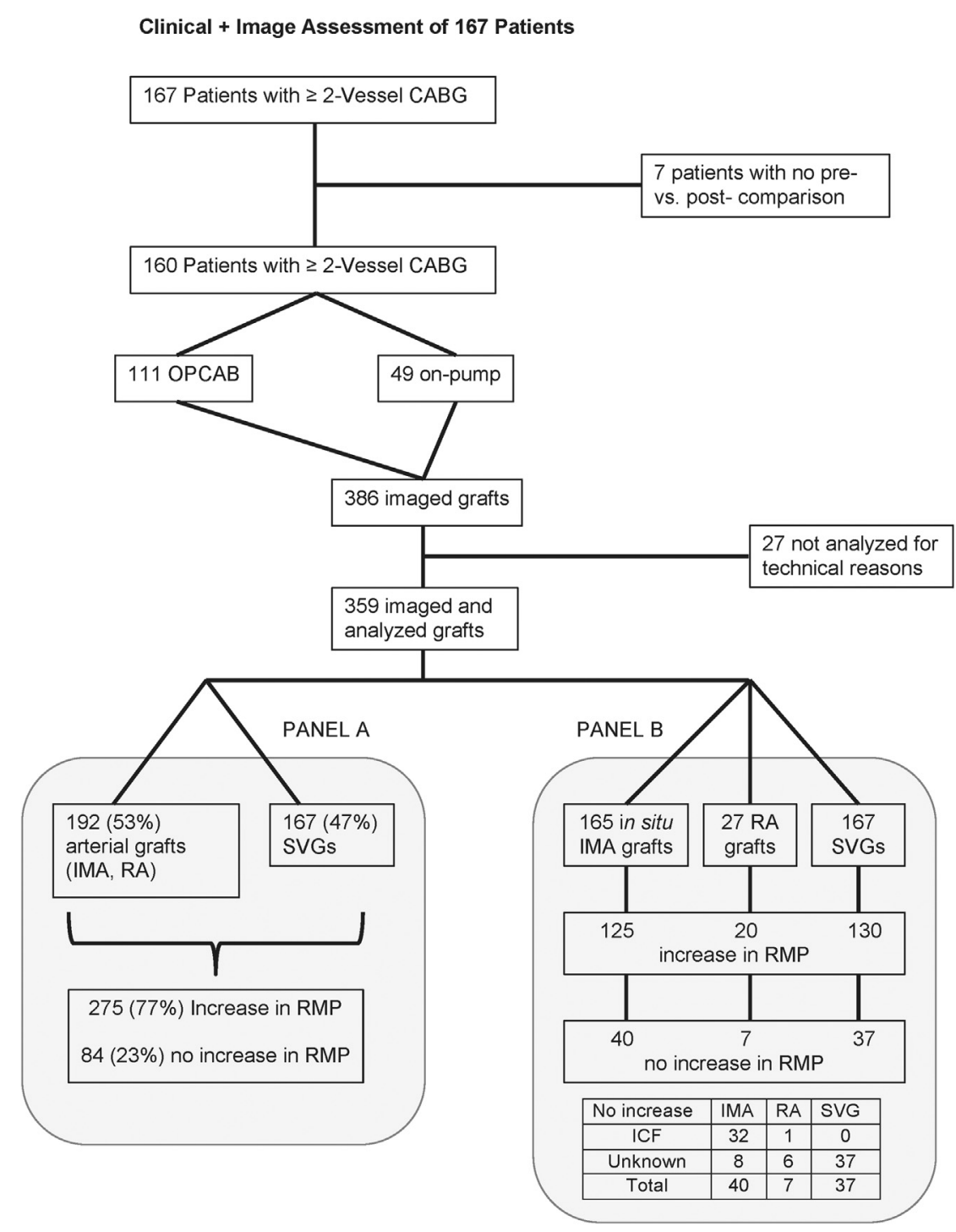

FIGURE 3. Diagram illustrating the clinical and imaging flow of the study. $C A B G$, Coronary artery bypass grafting; $O P C A B$, off-pump CABG; $I C F$, image-described competitive flow; IMA, internal mammary artery; $R A$, radial artery; $S V G$, saphenous vein graft; $R M P$, regional myocardial perfusion, as assessed using near-infrared fluorescence complex angiography and perfusion analysis.

Second, correlation was absent between the changes in RMP and documented perfusion deficits and regional ischemia preoperatively in these patients. This correlation would confirm that the change in RMP with grafting was a physiologic response to that perfusion deficit or ischemia. Several studies, including the FAME 3 study and the National Heart, Lung, and Blood Institute Cardiac Surgical Network FFR-CABG Trial, are being designed to specifically leverage this preoperative functional anatomy with functional outcomes. Intraoperative documentation of the physiologic consequences of revascularization on a per graft basis might be useful in these circumstances.

Third, the present clinical experience was mostly accrued from patients undergoing either OPCAB or beating heart CABG with CPB support. Although this created the enormous opportunity to study revascularization physiology in real time, additional study is necessary to determine whether these beating heart results can be demonstrated after CABG with cardioplegic arrest.

B, No increase occurred in regional myocardial perfusion, despite widely patent bypass grafts beyond $\sim 75 \%$ stenoses according to the near-infrared fluorescence complex angiography and perfusion analysis evaluation. The patient whose data are shown in Figure A had a preoperative left anterior descending coronary artery fractional flow reserve of 0.73 , and the patient whose data are shown in part B had a preoperative fractional flow reserve of 0.81 . 


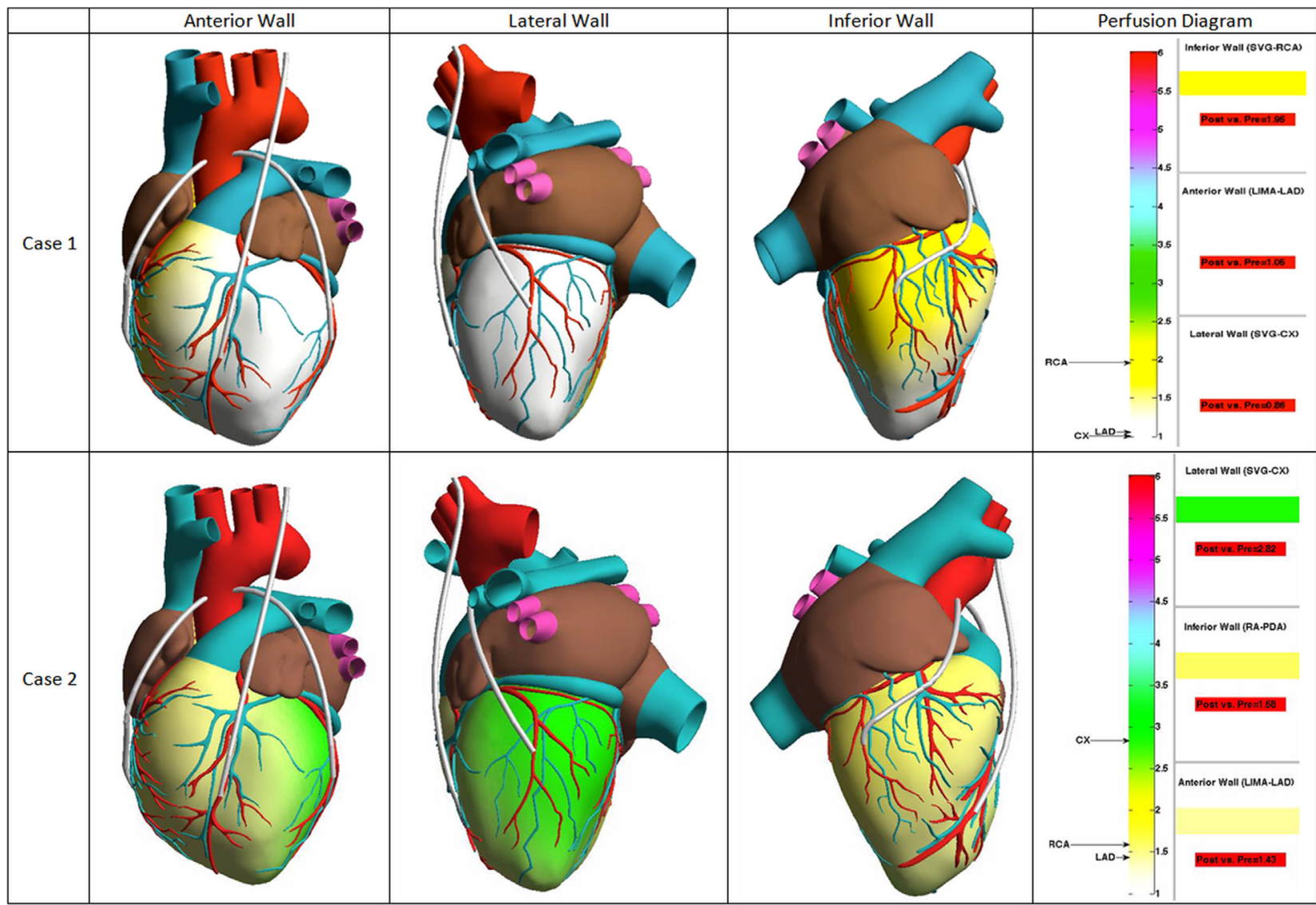

FIGURE 4. The individual regional myocardial perfusion data for 2 patients who had undergone multivessel off-pump coronary artery bypass grafting were aggregated into a 2-dimensional representation of the global change in perfusion in the heart as a result of bypass grafting. In case 1, only the inferior wall region had an increase in myocardial perfusion with grafting. In case 2, however, a significant change in perfusion occurred in all 3 areas as a result of revascularization. In both cases, all grafts were widely angiographically patent as documented by the near-infrared fluorescence angiographic evaluation. The difference between these 2 patients' physiologic outcomes with bypass grafting highlight the importance of a new knowledge opportunity inherent in intraoperative physiologic evaluation of both angiography and perfusion at bypass grafting. $C X$, Circumflex region; $L A D$, left anterior descending region; LIMA-LAD, left internal mammary artery to left anterior descending; $R C A$, right coronary artery region; $R A-P D A$, right coronary-posterior descending artery; $S V G-C X$, saphenous vein graft to circumflex marginal; $S V G-R C A$, saphenous vein graft to right coronary artery.

Finally, what we do not know is both our greatest limitation and opportunity. The experience accrued from imaging almost 1000 patients and some 3000 grafts with NIRF-CAPA has confirmed that a better understanding of the physiology of surgical revascularization requires sophisticated intraoperative technologies that can directly link imaging to the underlying physiology. At present, the most comprehensive representation of the angiographic and functional consequences of surgical revascularization on a per-graft based is with the NIRF-CAPA platform technology we used in the present study.

\section{CONCLUSIONS}

We have presented the initial experience documenting that the nature of the TVECA stenosis is important in determining the imaging-based physiologic consequences of CABG, on a per graft basis. The information imbedded in real-time intraoperative imaging at $\mathrm{CABG}$ promises to improve the physiologic understanding and outcomes of surgical revascularization in the FFR era.

\section{References}

1. Favaloro RG. Critical Analysis of Coronary Artery Bypass Graft Surgery: A 30-Year Journey. J Am Coll Cardiol. 1998;31(Suppl B):1B-63B.

2. Topol EJ, Nissen SE. Our preoccupation with coronary luminology: the dissociation between clinical and angiographic findings in ischemic heart disease. Circulation. 1995;92:2333-42.

3. Buda AJ, MacDonald IL, Anderson MH, Strauss HD, David TE, Berman ND. Long-term results following coronary bypass operation: importance of preoperative actors and complete revascularization. J Thorac Cardiovasc Surg. 1981;82:383-90.

4. Ferguson TB Jr, Hammill BG, Peterson ED, DeLong ER, Grover FL. A decade of change-risk profiles and outcomes for isolated coronary artery bypass grafting procedures, 1990-1999: a report from the STS National Database Committee and the Duke Clinical Research Institute. Society of Thoracic Surgeons. Ann Thorac Surg. 2002;73:480-9; discussion 489-90.

5. ElBardissi AW, Aranki SF, Sheng S, O'Brien SM, Greenberg CC, Gammie JS. Trends in isolated coronary artery bypass grafting: an analysis of the Society of Thoracic Surgeons adult cardiac surgery database. J Thorac Cardiovasc Surg. 2012;143:273-81. 
6. Ben-Gal Y, Mohr R, Uretzky G, Medalion B, Hendler A, Hansson N, et al. Drugeluting stents versus arterial myocardial revascularization in patients with diabetes mellitus. J Thorac Cardiovasc Surg. 2006;132:861-6.

7. Farkouh ME, Domanski M, Sleeper LA, Siami FS, Dangas G, Mack M, et al. Strategies for multivessel revascularization in patients with diabetes. $N$ Engl J Med. 2012;367:2375-84

8. Fearon WF, Bornschein B, Tonino PA, Gothe RM, Bruyne BD, Pijls NH, et al. Economic evaluation of fractional flow reserve-guided percutaneous coronary intervention in patients with multivessel disease. Circulation. 2010;122:2545-50.

9. Boden WE, O'Rourke RA, Teo KK, Hartigan PM, Maron DJ, Kostuk WH, et al, for the COURAGE Trial Research Group. Optimal medical therapy with or without PCI for stable coronary disease. N Engl J Med. 2007;356:1-14.

10. Shaw LJ, Berman DS, Maron DJ, Mancini GB, Hayes SW, Hartigan PM, et al. Optimal medical therapy with or without percutaneous coronary intervention to reduce ischemic burden: results from the Clinical Outcomes Utilizing Revascularization and Aggressive Drug Evaluation (COURAGE) trial nuclear substudy. Circulation. 2008;117:1283-91.

11. Pijls NH, Fearon WF, Tonino PA, Siebert U, Ikeno F, Bornschein B, et al. Fractional flow reserve versus angiography for guiding percutaneous coronary intervention in patients with multivessel coronary artery disease: 2-year follow-up of the FAME (Fractional Flow Reserve Versus Angiography for Multivessel Evaluation) study. J Am Coll Cardiol. 2010;56:177-84.

12. Tonino PA, De Bruyne B, Pijls NH, Siebert U, Ikeno F, van't Veer M, et al. Fractional flow reserve versus angiography for guiding percutaneous coronary intervention. N Engl J Med. 2009;360:213-24.

13. De Bruyne B, Pijls NH, Kalesan B, Barbato E, Tonino PA, Piroth Z, et al. Fractional flow reserve-guided PCI versus medical therapy in stable coronary disease. N Engl J Med. 2012;367:991-1001.

14. Balacumaraswami L, Taggart DP. Digital tools to facilitate intraoperative coronary artery bypass graft patency assessment. Semin Thorac Cardiovasc Surg. 2004;16:266-71

15. Balacumaraswami L, Abu-Omar Y, Anastasiadis K, Choudhary B, Pigott D, Yeong SK, et al. Does off-pump total arterial grafting increase the incidence of intraoperative graft failure? J Thorac Cardiovasc Surg. 2004;128:238-44.

16. Singh SK, Desai ND, Chikazawa G, Tsuneyoshi H, Vincent J, Zagorski BM, et al. The Graft Imaging to Improve Patency (GRIIP) clinical trial results. J Thorac Cardiovasc Surg. 2010;139:294-301.e1.

17. Takahashi M, Ishikawa T, Higashidani K, Katoh H. SPY: an innovative intra-operative imaging system to evaluate graft patency during off-pump coronary artery bypass grafting. Interact Cardiovasc Thorac Surg. 2004;3:479-83.

18. Waseda K, Ako J, Hasegawa T, Shimada Y, Ikeno F, Ishikawa T, et al. Intraoperative fluorescence imaging system for on-site assessment of off-pump coronary artery bypass graft. JACC. 2009;2:604-12.

19. Balacumaraswami L, Abu-Omar Y, Choudhary B, Pigott D, Taggart DP. A comparison of transit-time flowmetry and intraoperative fluorescence imaging for assessing coronary artery bypass graft patency. $J$ Thorac Cardiovasc Surg. 2005; 130:315-20.

20. Alander JT, Kaartinen I, Laakso A, Pätilä T, Spillman T, Tuchin VV, et al. A review of indocyanine green fluorescent imaging in surgery. Int $J$ Biomed Imaging. 2012;2012:940585.

21. Unno N, Suzuki M, Yamamoto N, Inuzuka K, Sagara D, Nishiyama M, et al. Indocyanine green fluorescence angiography for intraoperative assessment of blood flow: a feasibility study. Eur J Vasc Endovasc Surg. 2008;35:205-7.

22. Detter C, Wipper S, Russ D, Iffland A, Burdorf L, Thein E, et al. Fluorescent cardiac imaging: a novel intraoperative method for quantitative assessment of myocardial perfusion during graded coronary artery stenosis. Circulation. 2007; 116:1007-14

23. Polomsky M, He X, O'Brien SM, Puskas JD. Outcomes of off-pump versus on-pump coronary artery bypass grafting: impact of preoperative risk. $J$ Thorac Cardiovasc Surg. 2013;145:1193-8.

24. Dauerman HL. Reasonable incomplete revascularization. Circulation. 2011;123: 2337-40.

25. Kim YH, Ahn JM, Park DW, Song HG, Lee YH, Kim WJ, et al. Impact of ischemia-guided revascularization with myocardial perfusion imaging for patients with multivessel coronary disease. J Am Coll Cardiol. 2012;60:181-90.

26. Weintraub WS. Should ischemia guide revascularization? J Am Coll Cardiol. 2012;60:191-2

27. Kern M. Limitations of angiographic predictors of bypass graft patency. $J A m$ Coll Cardiol. 2008;52:885-8.

28. Botman CJ, Schonberger J, Koolen S, Penn O, Botman H, Dib N, et al. Does stenosis severity of native vessels influence bypass graft patency?
A prospective fractional flow reserve-guided study. Ann Thorac Surg. 2007;83: 2093-7.

29. Sabik JF III. Understanding saphenous vein graft patency. Circulation. 2011;124 273-5

30. Sabik JF III, Blackstone EH. Coronary artery bypass graft patency and competitive flow. J Am Coll Cardiol. 2008;51:126-8.

31. Alexander JH, Hafley G, Harrington RA, Peterson ED, Ferguson TB Jr, Lorenz TJ, et al. Efficacy and safety of edifoligide, an E2F transcription factor decoy, for prevention of vein graft failure following coronary artery bypass graft surgery: PREVENT IV: a randomized controlled trial. JAMA. 2005;294: 2446-54.

32. Lopes RD, Williams JB, Mehta RH, Reyes EM, Hafley GE, Allen KB, et al Edifoligide and long-term outcomes after coronary artery bypass grafting: PRoject of Ex-vivo Vein graft ENgineering via Transfection IV (PREVENT IV) 5-year results. Am Heart J. 2012;164:379-86.e1.

33. Kern MJ, Samady H. Current concepts of integrated coronary physiology in the catheterization laboratory. J Am Coll Cardiol. 2010;55:173-85.

34. Botman K-J, Piljs NHJ, Bech JW, Aarnoudse W, Peels K, van Straten B, et al. Percutaneous coronary intervention or bypass surgery in multivessel disease? A tailored approach based on coronary pressure measurement. Cathet Cardiovasc Intervent. 2004;63:184-91.

\section{Discussion}

Dr Joseph F. Sabik (Cleveland, Ohio). I would like to congratulate Dr Ferguson and colleagues on a fine presentation and study and thank them for providing me with a copy of their report in advance of the meeting.

As we heard, the authors used NIRF-CAPA to evaluate the functional importance of coronary artery stenosis by comparing myocardial perfusion before and after CABG. They found that despite significant coronary artery stenosis by angiography, bypass grafting did not improve myocardial perfusion in slightly $>20 \%$ of the territories grafted, suggesting to the authors that these stenoses were not functionally significant and therefore did not need grafting and that grafting them might have led to harm. I have a few questions for the authors.

My first question has to do with the usefulness of this technique. Whether coronary artery stenosis is physiologically significant is important to know before grafting. With this technique, the functional significance of the stenosis is determined only after the artery has been grafted. How can this technique guide in decision-making as the title of your presentation suggests?

My second question has to do with whether measuring changes in myocardial perfusion without stress is an accurate method of determining the functional significance of a coronary blockage. The physiologic test used to determine the functional importance of coronary artery stenosis is to provoke the myocardium with stress. For instance, during the measurement of a functionally significant coronary stenosis using FFR, a vasodilator, such as adenosine, is given to induce hyperemia before measuring the decrease in perfusion across the stenosis. In your measurement of myocardial perfusion, no stressing of the myocardium has been done either before or after grafting. For example, for the procedures done on pump, the blood flow measurements were made with the patient still on cardiopulmonary bypass, a very low workload on the myocardium. Is it not possible that this method might have underestimated the functional significance of the coronary stenosis?

My third question is, because of studies such as FAME 1 and FAME 2, the importance of not stenting nonphysiologic 
significant coronary artery stenosis has been demonstrated. In your report, you extrapolated these findings to also apply to coronary artery bypass grafting. These 2 revascularization techniques are very different, and one could hypothesize that grafting a nonphysiologic stenosis might result in only the graft closing, with no resultant harm to the patient. In your report, you showed excellent results for the entire group of patients. Did you compare the outcomes in the patients with grafts that did not increase myocardial perfusion with those with grafts that did increase myocardial perfusion, and if you did, were there any differences?

My final question has to do with the FFR data that were collected preoperatively for some patients. Were you able to determine whether the FFR data you had correlated with your findings of myocardial perfusion after grafting?

I would once again like to congratulate the authors for an interesting study and thank the Association for the opportunity of discussing this report.

Dr Ferguson. Thank you, Dr Sabik, and thank you for your comments.

After 50 years, the anatomic basis for CABG is changing. We are moving to an era in which the functionality of target vessel coronary artery stenoses are going to be characterized in the catheterization laboratory before patients come to surgery. In fact, we are going to be faced with a situation in which the cardiologists tell us to graft this stenosis based on functionality but do not graft this stenosis based on nonfunctionality. FFR introduces physiology into the catheterization laboratory, and we need a method in the operating room to be able to evaluate the physiologic response to revascularization and to validate the preoperative results to be able to determine that a patient has received the full benefit of this operation. This physiologic evolution certainly seems to be the trend and was the genesis for the National Heart, Lung, Blood Institute Cardiac Surgical Network FFR-guided CABG trial that is under discussion and other trials such as FAME 3 that are under discussion.

With regard to your second question, this is definitely not a stress evaluation of the myocardium, unlike FFR. This evaluation is done during a 90 -second period in which the heart rate, blood pressure, and coronary autoregulation are stable to obtain both the pregrafting image and the postgrafting image. We spent several years developing and validating that this is the case. Thus, what you see is a direct comparison, which is the difference in flow down the epicardial coronary versus the epicardial coronary plus the graft, and the consequent effect on myocardial perfusion. Currently, the only correlation with the maximal vasodilation circumstance with FFR is that $23 \%$ of these imaged grafts do not show a regional myocardial perfusion increase, $20 \%$ of the anatomic lesions between $71 \%$ and $90 \%$ in FAME did not have functionality associated with them, and $20 \%$ to $25 \%$ of the grafts that we sew have been occluded silently at 1 year, as determined by protocol-driven angiography in most of our major studies.

As to your third question, we do not yet have formal long-term follow-up on the fate of the grafts without an increase in perfusion. In these multivessel CABG patients, however, essentially all patients in the group had some measured increase in regional perfusion. At the other end of the spectrum, the number of patients with a substantial increase in all regions was relatively small, approximately $15 \%$ to $20 \%$. The short-term outcomes were excellent, and the absence of a perfusion increase on a per graft basis was not associated with acute perioperative events based on the present analysis.

Addressing your fourth question, we do have data, not yet published, considering the correlation of the regional myocardial perfusion change, anatomy, and myocardial ischemia. When perfusion change is compared with the SYNTAX-characterized anatomy alone, the correlation was not statistically significant. If one adds the functionality of the myocardium, from the FFR, preoperative magnetic resonance imaging level, or clinical status, that relationship becomes highly significant, suggesting that the measurement of regional myocardial perfusion change at surgery is an index of the relief of ischemia or the relief of the perfusion deficit measured preoperatively in these patients.

Dr Sabik. I would just like to add a word of caution about FFR-guided surgical revascularization. Some studies have now shown that if one grafts a vessel that has an FFR $>8$, only 1 of 5 of those grafts are going to close; 8 of the 10 , or $80 \%$, are going to remain open; and we know that the vessels that cause myocardial infarction are those moderate lesions that thrombose. When we consider surgery, we are thinking about a long-term solution to a problem, and those $80 \%$ of grafts might very well be protective over the long run. Also, when we think about FFR and stenting, it is really owing to the periprocedural complications and the restenosis why stenting does not work. Finally, I just worry that if we extrapolate the findings of FAME to surgery, we might be doing $80 \%$ of our patients harm.

Dr Ferguson. I actually completely agree with you, Joe. This study is not advocating adopting an FFR-guided strategy for revascularization. It is advocating imaging of the physiologic consequences of revascularization in the operating room to learn how we can improve CABG from a physiologic perspective, beyond using the anatomy alone.

For example, the late mortality from FREEDOM and SYNTAX cannot be explained by the anatomy, because both arms of the intervention in both of those trials were anatomically based. Thus, something else besides anatomy must be driving the longterm survival benefit in those patients. Our data suggest that it might be the functional response to bypass surgery that we have been creating all along. Understanding the cause of this benefit will be important in the evolving, physiologic-based revascularization era beyond anatomy alone.

Dr John H. Calhoon (San Antonio, Tex). Elegant study. To continue with what Dr Sabik said, how are you going to use this technique to help us know how to do CABG or more appropriate CABG when we are considering the 10-year survival for the operating room as opposed to the 10-minute outcome in the catheterization laboratory? I am afraid that this technology falls into the same category that Joe described, we are considering use of a technology designed to help cardiologists know when to intervene on a lesion rather than to help us know what to do for a patient long term. In other words, how might this technology be applied successfully in CABG?

Dr Ferguson. The new window here is a window of opportunity into the physiology of revascularization that we accomplish in the operating room. 
For example, to be able to directly visualize competitive flow in bypass grafts is potentially a very real asset in our ability to learn what the influence of competitive flow on long-term graft survival actually is, because right now we do not know.

Using the traditional anatomic construct for CABG, we do a number of tasks based on assumptions but not real data, because we have never measured it in the operating room.
As I stated, the idea that something other than anatomy is what drives the long-term survival from SYNTAX and FREEDOM trials might be directly linked to what we create functionally at surgery, which is a safety net of perfusion. Linking the preoperative functional anatomy and long-term outcomes will greatly be facilitated by the intraoperative assessment of the physiologic consequences of revascularization, which could be the physiologic substrate for long-term survival benefit in CABG. 


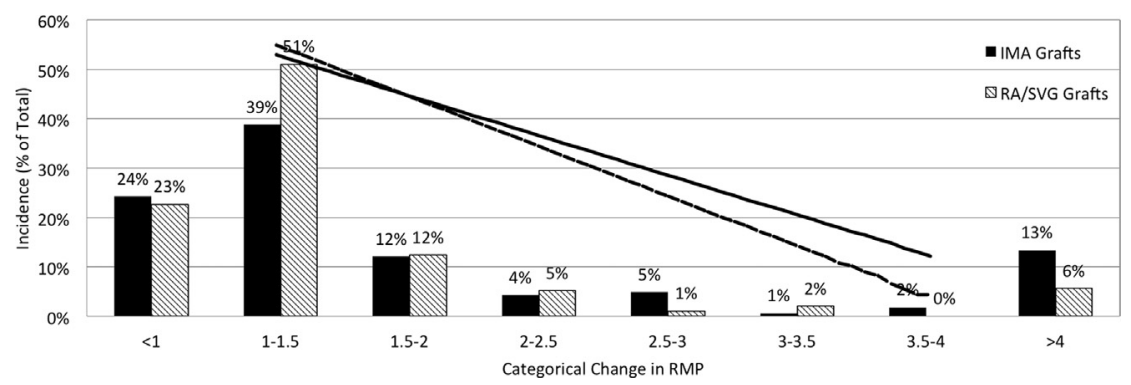

FIGURE E1. Histogram plot of the percentage of grafts with a categorized magnitude of change in regional myocardial perfusion (RMP) as a result of bypass grafting. Black bars indicate in situ internal mammary artery (IMA) grafts; hashed bars, aortocoronary (saphenous vein graft [SVG]/radial artery $[R A]$ ) grafts. The straight lines represent the log exponential decay curves for the IMA (solid black line) and aortocoronary grafts (dashed line). The slopes of the 2 lines differed by $25 \%(P<.05)$. More clustering of smaller increases $(10 \%$ to $75 \%,<2.0)$ was found in the aortocoronary grafts, and the IMA grafts ( $92 \%$ to the left anterior descending coronary artery) had a broader range of RMP increases. 